\title{
Candida SPP. Colonization in NICU: A 2-Year Surveillance Study
}

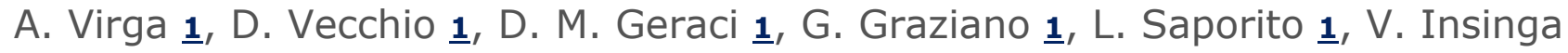

$\underline{\mathbf{1}}$, C. M. Maida $\underline{\mathbf{1}}$, C. Mammina $\underline{\mathbf{1}}, \mathrm{M}$. Giuffrè $\underline{\mathbf{1}}$

1Department of Sciences for the Health Promotion and Mother and Child Care

"G. D'Alessandro," Hygiene Section, University of Palermo, Palermo, Italy Congress Abstract

Presenter: A.Virga (e-mail: anto.virga@gmail.com)

Introduction: Fungal infections are an important cause of increased morbidity and mortality in infants admitted to neonatal intensive care units (NICUs). In VLBW infants, Candida (C.) albicans is the third most common cause of neonatal late onset sepsis (LOS). The overall incidence of candidemia in NICU is increasing because of the longer survival and the invasive procedures related with the intensive care of extremely preterm infants. Prevention of candidemia in neonates is supported by the identification and adequate management of specific risk factors, including low birth weight, use of invasive devices, prolonged hospitalization and use of broad-spectrum antimicrobial agents. Effective prophylactic strategies have recently become available, but the identification of the best possible strategies to manage high-risk infants is still a priority. Prior colonization is a key risk factor for candidemia. For this reason, surveillance studies to monitor incidence, species distribution, and antifungal susceptibility profiles are mandatory.

Materials and Methods: In 2013 and 2014, we performed a cohort, prospective surveillance study in our NICU, collecting weekly nasal and rectal swabs. For each patient, clinical and demographic data expected to affect Candida colonization were recorded. We evaluated Candida spp. colonization rate and assessed the related risk factors. Swabs were placed on Sabouraud agar and incubated at $30^{\circ} \mathrm{C}$ for 4 days. Candida growth on agar was confirmed by microscopic observation. Moreover, Candida spp. were identified through Candida chromogenic agar (ChromAgar Candida, Laboratorios Conda) and API $® 20 C$ AUX (Biomérieux). Statistical analysis was performed by EpiInfo (CDC software) using the chi square or the Fisher's exact method, when indicated. We assumed as statistically significant a $p$-value $<0.05$.

Results: In this 2-year study, we enrolled 520 patients and we analyzed 1,259 nasal and 1,255 rectal swabs. From 472 out of 520 patients we collected complete microbiological, clinical and demographic data. 48 out of 472 $(10.17 \%)$ patients tested positive for Candida spp. at least once. In particular, 26 patients tested positive for C. albicans, 16 for C. parapsilosis, 6 for $C$. glabrata and 1 each for $C$. guilliermondii and an environmental mold. All the colonized patients had their rectal samples positive, and 7 their nasal samples as well. 15 patients out of $472(3.18 \%)$ had more than one rectal or nasal swab positive during their NICU stay. Importantly, 9 patients out of 15 tested 
negative at the first sampling, suggesting that they have acquired Candida spp. colonization during their stay. Table 1 summarizes data about risk factors for Candida colonization in the patients under study. No systemic infection by Candida spp. was reported during the study.

Conclusion: Our experience suggest that an effective microbiological surveillance can allow for implementing proper, effective and timely control measures in a high-risk setting.

Keywords: candida, surveillance 